\title{
A VEHICLE-TRACK-SOIL DYNAMIC INTERACTION PROBLEM IN SEQUENTIAL AND PARALLEL FORMULATION
}

\author{
JANUSZ KOGUT, HENRYK CIUREJ \\ Cracow University of Technology, Warszawska 22, 31-155 Cracow, Poland \\ email: jkogut@pk.edu.pl, hciurej@usk.pk.edu.pl
}

\begin{abstract}
Some problems regarding numerical modeling of predicted vibrations excited by railway traffic are discussed. Model formulation in the field of structural mechanics comprises a vehicle, a track (often in a tunnel) and soil. Time consuming computations are needed to update large matrices at every discrete step. At first, a sequential Matlab code is generated. Later on, the formulation is modified to use grid computing, thereby a significant reduction in computational time is expected.
\end{abstract}

Keywords: numerical modeling, identification, vibrations prediction.

\section{Introduction}

Dynamic prediction of the vibration excited by trains running on a railway track is a very important issue nowadays. This type of vibration analysis is crucial since the residents in the vicinity of existing and newly constructed tram and underground lines are becoming more annoyed by rolling stock generated dynamic effects (Cox and Wang, 2003). An accurate model formulation in the field of structural mechanics comprises a vehicle, a track (a tunnel) and soil.

Several problems of a theoretical-numerical model used to predict vibrations excited by railway traffic are discussed below. This model takes into consideration transfer functions between the track lying either on the subgrade or in the underground tunnel and a free field. The train can be modelled as a multi-body vehicle chain containing several wagons (Kogut and Ciurej, 2005a). Every wagon may consist of a body and bogies. Every bogie has two axles and four wheels. A primary suspension is located between the axle and the bogie, while a secondary suspension is constructed between the bogie and the body. The dynamic parameters of rolling stock were identified earlier (Kogut and Ciurej, 2006). The subway wagon was modelled in a similar way and the structural parameters, present in the model matrices, are being indentified (Kogut and Ciurej, 2008).

Every wheel is characterized by its own irregularity, which may be measured or estimated. As far as the track is concerned, the irregularities of both railheads differ and can be measured or estimated as well (Steenbergen, 2008). Dynamic axle loads due to the rail and wheel irregularities may be evaluated (Kogut and Ciurej, 2005b). A single point wheel-rail contact is assumed in the model. As for the dynamic compliance, track and tunnel transfer functions may be computed or measured (Gupta et al., 2007).

Contact forces between the rail and the wheels constitute the loads, which are transferred to the environment through the track (the tunnel) and propagate in the soil. The entire model may be validated by means of advanced "in-situ" measurements.

\section{Numerical prediction model}

A single wagon (Fig. 1) consists of a body and two bogies, each of them supported by two axles. The equation of motion of the rolling stock can be given as follows:

$$
\mathbf{M} \ddot{\mathbf{x}}(t)+\mathbf{C} \dot{\mathbf{x}}(t)+\mathbf{K} \mathbf{x}(t) \mathbf{Q}-\mathbf{R}(t) .
$$

It seems to be important to subdivide the degrees of freedom (DOF) into those dedicated to the boundary, i.e., contact points between the wheel and the railhead and the internal degrees of freedom, which are related to the vehicle structure. Equations of motion of such a vehicle are 


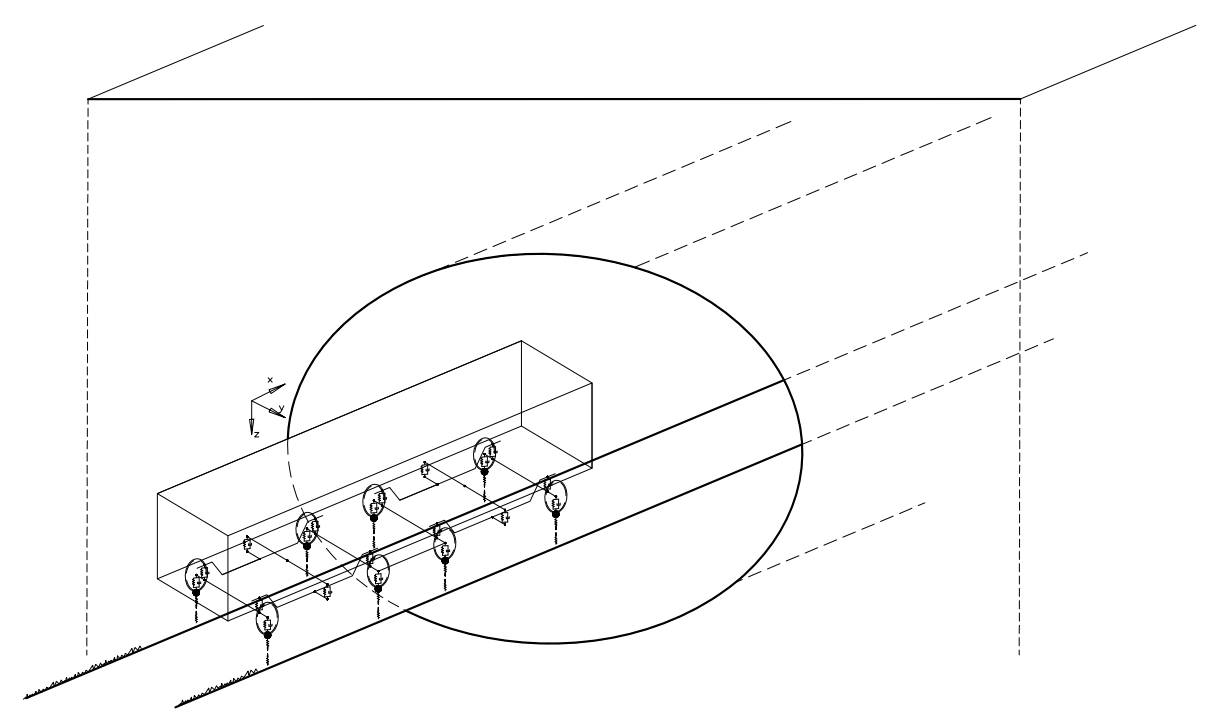

Fig. 1. Wagon on a track in a tunnel.

given (Kogut and Ciurej, 2005b) as follows:

$$
\begin{gathered}
{\left[\begin{array}{ll}
\mathbf{M}_{11} & \mathbf{M}_{12} \\
\mathbf{M}_{21} & \mathbf{M}_{22}
\end{array}\right]\left[\begin{array}{l}
\ddot{\mathbf{x}}_{1} \\
\ddot{\mathbf{x}}_{2}
\end{array}\right]+\left[\begin{array}{ll}
\mathbf{C}_{11} & \mathbf{C}_{12} \\
\mathbf{C}_{21} & \mathbf{C}_{22}
\end{array}\right]\left[\begin{array}{l}
\dot{\mathbf{x}}_{1} \\
\dot{\mathbf{x}}_{2}
\end{array}\right]} \\
+\left[\begin{array}{ll}
\mathbf{K}_{11} & \mathbf{K}_{12} \\
\mathbf{K}_{21} & \mathbf{K}_{22}
\end{array}\right]\left[\begin{array}{l}
\mathbf{x}_{1} \\
\mathbf{x}_{2}
\end{array}\right]=\left[\begin{array}{l}
\mathbf{Q}_{1} \\
\mathbf{Q}_{2}
\end{array}\right]-\left[\begin{array}{c}
\mathbf{0} \\
\mathbf{R}_{2} \cdot
\end{array}\right]
\end{gathered}
$$

(For simplicity, the time relationship is dropped.) One may notice from Eqn. (2) that index 1 is dedicated to the internal DOF and index 2 to the external DOF. Moreover, in (1) and (2), the symbols are as follows:

x: generalized displacement vector of dimension $N_{P} \times$ 1 ; generalized vehicle velocity and acceleration vectors are $\dot{\mathbf{x}}$ and $\ddot{\mathbf{x}}$, respectively. Vector $\mathbf{x}_{1}$ is of dimension $N \times 1$ and vector $\mathbf{x}_{2}$ of $N_{R} 1$; the following relation is satisfied:

$$
\mathbf{x}=\left[\begin{array}{l}
\mathbf{x}_{1} \\
\mathbf{x}_{2}
\end{array}\right]
$$

while the number of internal DOFs, is $N=N_{P}-$ $N_{R}$. The internal DOF vector $\mathbf{x}_{2}$ exhibits a kinematic excitation and can be written as follows:

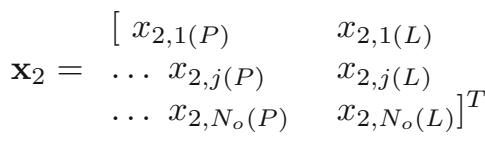

where $N_{o}$ is the number of axles.
M: (symmetric) $N_{P} \times N_{P}$ mass matrix, of the form

$$
\mathbf{M}=\left[\begin{array}{ll}
\mathbf{M}_{11} & \mathbf{M}_{12} \\
\mathbf{M}_{21} & \mathbf{M}_{22}
\end{array}\right],
$$

where $\mathbf{M}_{11}$ is of dimension $N \times N, \mathbf{M}_{22}$ is of dimension $N_{R} \times N_{R}, \mathbf{M}_{12}$ is of dimension $N \times N_{R}$. By symmetry, $\mathbf{M}=\mathbf{M}^{T}$ and $\mathbf{M}_{12}=\mathbf{M}_{21}^{T}$. The mass matrix $\mathbf{M}$ may be diagonal and then $\mathbf{M}_{12}=\mathbf{0}$. It should be pointed out that the mass matrix $\mathbf{M}$ consists not only of the vehicle masses but also of additional masses (such as passengers and luggage) due to the operational loads.

C: (symmetric) damping matrix, which can be written as

$$
\mathbf{C}=\left[\begin{array}{ll}
\mathbf{C}_{11} & \mathbf{C}_{12} \\
\mathbf{C}_{21} & \mathbf{C}_{22}
\end{array}\right]
$$

By symmetry, $\mathbf{C}=\mathbf{C}^{T}$ and $\mathbf{C}_{12}=\mathbf{C}_{21}^{T}$ and the dimensions are the same as those of the mass matrices. The damping matrix cannot be applied as a proportional one (i.e., $\mathbf{C}=\alpha \mathbf{M}+\beta \mathbf{K}$ ) due to the discrete elements.

$\mathbf{K}$ : (symmetric) stiffness matrix, which can be written as

$$
\mathbf{K}=\left[\begin{array}{ll}
\mathbf{K}_{11} & \mathbf{K}_{12} \\
\mathbf{K}_{21} & \mathbf{K}_{22}
\end{array}\right]
$$

$\mathbf{K}=\mathbf{K}^{T}$ and $\mathbf{K}_{12}=\mathbf{K}_{21}^{T}$ and the dimensions are the same as those of the mass matrices. Matrix $\mathbf{K}$ is singular and $\operatorname{det}(\mathbf{K})=0$, but $\operatorname{det}\left(\mathbf{K}_{i i}\right) \neq 0$ because 
submatrices $\mathbf{K}_{i i}$ comply with the boundary conditions.

Q: $N_{P} \times 1$ vector of constant static forces including the operational forces is defined as follows:

$$
\mathbf{Q}=\left[\begin{array}{l}
\mathbf{Q}_{1} \\
\mathbf{Q}_{2}
\end{array}\right]
$$

where $\mathbf{Q}_{1}$ and $\mathbf{Q}_{2}$ have dimensions $N \times 1$ and $N_{R} \times 1$, respectively.

R: $N_{P} \times 1$ dynamic reaction vector:

$$
\mathbf{R}=\left[\begin{array}{c}
\mathbf{0} \\
\mathbf{R}_{2}
\end{array}\right]
$$

where $\mathbf{R}_{2}$ is $N_{R} \times 1$, resulting in:

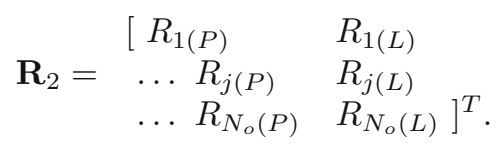

Even elements are due to the left rail $(L)$ interaction, while the odd ones follow the right rail $(P)$. The rear axle is first.

After the reformulation of Eqn. (2) and extracting $\mathbf{R}_{2}$, the equations of motion are re-arranged as

$$
\left\{\begin{array}{c}
\mathbf{M}_{11} \ddot{\mathbf{x}}_{1}+\mathbf{C}_{11} \dot{\mathbf{x}}_{1}+\mathbf{K}_{11} \mathbf{x}_{1} \\
=\mathbf{Q}_{1}-\left(\mathbf{M}_{12} \ddot{\mathbf{x}}_{2}+\mathbf{C}_{12} \dot{\mathbf{x}}_{2}+\mathbf{K}_{12} \mathbf{x}_{2}\right) \\
\mathbf{R}_{2}=\mathbf{Q}_{2}-\left(\mathbf{M}_{21} \ddot{\mathbf{x}}_{1}+\mathbf{C}_{21} \dot{\mathbf{x}}_{1}+\mathbf{K}_{21} \mathbf{x}_{1}\right) \\
-\left(\mathbf{M}_{22} \ddot{\mathbf{x}}_{2}+\mathbf{C}_{22} \dot{\mathbf{x}}_{2}+\mathbf{K}_{22} \mathbf{x}_{2}\right) .
\end{array}\right.
$$

Note that the first equation of 11 is stationary and describes the motion of the body and the bogies. Vector $\mathbf{x}_{1}(t)$ contains an independent DOF. The right-hand side consists of static forces $\mathbf{Q}_{1}$ and the kinematic force $-\left(\mathbf{M}_{22} \ddot{\mathbf{x}}_{2}+\mathbf{C}_{22} \dot{\mathbf{x}}_{2}+\mathbf{K}_{22} \mathbf{X}_{2}\right)$ depending on a kinematic excitation represented as a vector $\mathbf{x}_{2}(t)$. If the vibrations of the vehicle occur around the equilibrium state $\hat{\mathbf{x}}_{1}$, then

$$
\mathbf{x}_{1}(t)=\tilde{\mathbf{x}}_{1}(t)+\hat{\mathbf{x}}_{1}, \quad \mathbf{R}_{2}(t)=\tilde{\mathbf{R}}_{2}(t)+\hat{\mathbf{R}}_{2},
$$

where $\tilde{\mathbf{x}}_{1}(t)$ is a fluctuation around $\hat{\mathbf{x}}_{1}$, and $\tilde{\mathbf{R}}_{2}(t)$ is a fluctuation of the reaction force with respect to the static force $\hat{\mathbf{R}}_{2}$. The derivatives are equal to

$$
\dot{\mathbf{x}}_{1}(t)=\dot{\tilde{\mathbf{x}}}_{1}(t), \quad \ddot{\mathbf{x}}_{1}(t)=\ddot{\tilde{\mathbf{x}}}_{1}(t) .
$$

Hence, for the static values and $\mathbf{x}_{2}=\mathbf{0}$ we have

$$
\left\{\begin{array}{l}
\mathbf{K}_{11} \hat{\mathbf{x}}_{1}=\mathbf{Q}_{1} \Rightarrow \hat{\mathbf{x}}_{1}=\mathbf{K}_{11}^{-1} \mathbf{Q}_{1} \\
\hat{\mathbf{R}}_{2}=\mathbf{Q}_{2}-\mathbf{K}_{21} \hat{\mathbf{x}}_{1} .
\end{array}\right.
$$

Using Eqns. (12) and (13), 111 may be written in the equilibrium state as

$$
\left\{\begin{aligned}
\mathbf{M}_{11} \ddot{\tilde{\mathbf{x}}}_{1}+\mathbf{C}_{11} \dot{\tilde{\mathbf{x}}}_{1}+\mathbf{K}_{11} \tilde{\mathbf{x}}_{1} & \\
& =-\left(\mathbf{M}_{12} \ddot{\mathbf{x}}_{2}+\mathbf{C}_{12} \dot{\mathbf{x}}_{2}+\mathbf{K}_{12} \mathbf{x}_{2}\right), \\
\tilde{\mathbf{R}}_{2}= & -\left(\mathbf{M}_{21} \ddot{\tilde{\mathbf{x}}}_{1}+\mathbf{C}_{21} \dot{\tilde{\mathbf{x}}}_{1}+\mathbf{K}_{21} \tilde{\mathbf{x}}_{1}\right) \\
& -\left(\mathbf{M}_{22} \ddot{\mathbf{x}}_{2}+\mathbf{C}_{22} \dot{\mathbf{x}}_{2}+\mathbf{K}_{22} \mathbf{x}_{2}\right) .
\end{aligned}\right.
$$

Equation (14) looks similar to Eqn. (11) but the static forces $\mathbf{Q}_{i}$ are eliminated. This implies that the vehicle vibrations are performed around the equilibrium state.

The kinematic excitation $\mathrm{x}_{2}$ consists of railhead irregularity $\mathbf{x}_{s z}$, wheel irregularity $\mathbf{x}_{w}$ and track vibration $\mathbf{u}_{2 t}$ due to the vehicular motion:

$$
\mathbf{x}_{2}=\mathbf{x}_{s z}+\mathbf{x}_{w}+\mathbf{u}_{2 t},
$$

where $\mathbf{u}_{2 t}$ depends on reaction $\mathbf{R}_{2}$ and its history as a convolution integral. Then $\mathbf{u}_{2 t}=\mathbf{u}_{2 t}\left(\mathbf{R}_{2}\right)$. The second equation of (11) constitutes the relationship between the vector of wheels' reactions $\mathbf{R}_{2}$ and the kinematic excitation $\mathbf{x}_{2}$, which is an implicit function of $\mathbf{R}_{2}$. In (11) both the equations are coupled not only through $\mathbf{x}_{1}$ and its derivatives but also by $\mathbf{x}_{2}$ and its derivatives and hence the dynamic reactions $\mathbf{R}_{2}(t)$.

In general, the stiffness matrix $\mathbf{K}$ may be non-linear and dependent on $\mathbf{x}$ as far as the material non-linearities are implemented, but this does not matter in this formulation, where the decoupling of the internal and external DOFs is introduced. The last is true when the model is discrete as in Eqn. (1). Moreover, the equations of motion (14) may be written as dynamic fluctuations around the equilibrium state, where matrix $\mathbf{K}_{11}$ is tangential (or secant) around the point of static equilibrium, although the point may be reached in a non-linear incremental way as for Eqn. (13), in which $\mathbf{K}_{11}$ is non-linear.

\section{Kinematic excitation due to rails and wheels}

The kinematic excitation of $x_{2}$ from Eqn. (15) is based on the railhead and wheel irregularities. The derivatives with respect to time depend on the translational velocity of the vehicle. The distance is a function of $v(t)$ :

$$
s(t)=\int_{0}^{t} v(\tau) \mathrm{d} \tau .
$$

The irregularities are functions of the distance. As for the railhead unevenness, $\mathbf{x}_{s z}=\mathbf{x}_{s z}\left[s(t)+\mathbf{s}_{0}\right]$, where $\mathbf{s}_{0}$ is the initial distance of the axle to the coordinate origin. The derivatives with respect to time are

$$
\begin{aligned}
& \dot{\mathbf{x}}_{s z}=\frac{\mathrm{d} \mathbf{x}_{s z}}{\mathrm{~d} s} v(t), \\
& \ddot{\mathbf{x}}_{s z}=\frac{\mathrm{d}^{2} \mathbf{x}_{s z}}{\mathrm{~d} s^{2}} v^{2}(t)+\frac{\mathrm{d} \mathbf{x}_{s z}}{\mathrm{~d} s} a(t),
\end{aligned}
$$


where $a(t)$ is the vehicle acceleration. In a similar way the derivatives of the wheel unevenness $\mathbf{x}_{k}$ with respect to time are described. Based on large databases, the description of irregularities may be done using a spectral density function (Van den Broeck, 2001). For $\boldsymbol{\Theta}_{n}=$ $2 \pi n_{n}\left(s-\mathbf{s}_{0}\right)-\theta_{n}$

$$
\mathbf{x}_{s z}(s)=\sqrt{2 \Delta n} \sum_{n=1}^{N_{i}} \sqrt{G\left(n_{n}\right)} \cos \left(\boldsymbol{\Theta}_{n}\right),
$$

where $n$ is a wave number [cycle/m], $\Delta n$ is the value dependent on the bandwidth of the wave numbers and $N_{i}$ intervals, $G(n)$ is a spectral density function of a railhead unevenness $\left[\mathrm{m}^{2} /\right.$ cycle $/ \mathrm{m}=\mathrm{m}^{3} /$ cycle $], \theta_{n}$ is a random phase $\theta_{n} \in[0,2 \pi]$.

As for the wheel unevenness, the function $R(\varphi)=$ $R_{0}+\Delta R(\varphi)$ may be an angular irregularity of the radius, and the relation between the distance and the wheel angle is $s(t)=\varphi(t) R_{0}$, where $R_{0}$ is the average wheel radius. Hence

$$
x_{k}(s(t))=\Delta R\left(\frac{s(t)}{R_{0}}\right) .
$$

The function may be expanded into a sine series:

$$
\Delta R(\varphi)=\sum_{k=1}^{N} r_{k} \sin \left(k \varphi+\varphi_{0 k}\right)
$$

where $r_{k}$ is the amplitude of unevenness, and $\varphi_{0 k}$ is the initial random phase. If there is no sliding, then $\mathbf{x}_{N}=$ $\mathbf{x}_{s z}+\mathbf{x}_{w}$, and the kinematic excitation is a sum of $\mathbf{x}_{2}=$ $\mathbf{x}_{N}+\mathbf{u}_{2 t}$

\section{Vehicle-track-soil interaction}

Dynamic soil response to every kind of excitation $G$ may be formulated as a convolution:

$$
u(\mathbf{x} ; t)=\int_{0}^{t} \int_{V} h(\mathbf{x}, \xi ; t-\tau) G(\xi ; \tau) \mathrm{d} \xi \mathrm{d} \tau,
$$

where $h(\mathbf{x}, \xi ; t-\tau)$ is the fundamental solution and $G(\xi ; \tau)$ are the dynamic forces due to excitations.

If the fundamental solutions (Green's functions in time and their time derivatives) are known at discrete points along the rail, as well as at the discrete points in the soil (or on the soil surface) as in Fig. 2, the problem is approximated at a limited number of points-nodes, due to the finite element (FEM) or other discrete solution method.

Shape functions are placed between points and the approximation of the response may be determined when the excitation is located between nodes. Here, linear shape functions $N_{1}(\eta)=-1 / l_{0} \eta+1$ and $N_{2}(\eta)=1 / l_{0} \eta$ are used, where $l_{0}$ is the element length and $\eta$ is the local coordinate of the element.
Finally, the vertical displacements of nodes on the left $\left(\mathbf{u}_{(L)}(t)\right)$ and right $\left(\mathbf{u}_{(P)}(t)\right)$ are represented by the following convolution:

$$
\begin{aligned}
{\left[\begin{array}{l}
\mathbf{u}_{(L)}(t) \\
\mathbf{u}_{(P)}(t)
\end{array}\right]=} & \int_{0}^{t} \underbrace{\left[\begin{array}{ll}
\mathbf{H}_{L}^{(L)}(t-\tau) & \mathbf{H}_{P}^{(L)}(t-\tau) \\
\mathbf{H}_{L}^{(P)}(t-\tau) & \mathbf{H}_{P}^{(P)}(t-\tau)
\end{array}\right]}_{\mathbf{H}(t-\tau)} \\
& \times\left[\begin{array}{l}
\mathbf{R}_{(L)}(\tau) \\
\mathbf{R}_{(P)}(\tau)
\end{array}\right] \mathrm{d} \tau
\end{aligned}
$$

where $\mathbf{R}_{(L)}(t)$ and $\mathbf{R}_{(P)}(t)$ are the reaction forces at nodes along the left and right rails, respectively, while $\mathbf{H}(t-\tau)$ is the matrix of fundamental solutions. The soil response at a surface point $A$ is given as a similar convolution:

$$
\begin{aligned}
\mathbf{u}_{A}(t)= & {\left[\begin{array}{l}
u_{(A x)}(t) \\
u_{(A y)}(t) \\
u_{(A z)}(t)
\end{array}\right] } \\
= & \int_{0}^{t} \underbrace{\mathbf{H}^{A}(t-\tau)}_{\left.\begin{array}{ll}
\mathbf{H}_{L}^{(A x)}(t-\tau) & \mathbf{H}_{P}^{(A x)}(t-\tau) \\
\mathbf{H}_{L}^{(A y)}(t-\tau) & \mathbf{H}_{P}^{(A y)}(t-\tau) \\
\mathbf{H}_{L}^{(A z)}(t-\tau) & \mathbf{H}_{P}^{(A z)}(t-\tau)
\end{array}\right]} \\
& \times\left[\begin{array}{l}
\mathbf{R}_{(L)}(\tau) \\
\mathbf{R}_{(P)}(\tau)
\end{array}\right] \mathrm{d} \tau .
\end{aligned}
$$

The dynamic response at every point of soil may be calculated for the forces generated between the wheel and the rail. The hunting wheelset does not influence vertical reactions. Wheel excitation takes into consideration the interactions between wheels in the wheelset and other wheels of a vehicle.

The responses regarding displacements, velocities and accelerations may be represented as

$$
\begin{aligned}
& \mathbf{u}_{(A)}(t) \\
& \quad=\int_{0}^{t} \mathbf{H}^{(A)}(t-\tau)\left[\begin{array}{cc}
\mathbf{Z}(\tau) & \mathbf{0} \\
\mathbf{0} & \mathbf{Z}(\tau)
\end{array}\right]\left[\begin{array}{l}
\mathbf{T}_{(L)} \\
\mathbf{T}_{(P)}
\end{array}\right] \mathbf{R}_{2}(\tau) \mathrm{d} \tau,
\end{aligned}
$$

$$
\begin{aligned}
& \mathbf{v}_{(A)}(t) \\
& =\int_{0}^{t} \dot{\mathbf{H}}^{(A)}(t-\tau)\left[\begin{array}{cc}
\mathbf{Z}(\tau) & \mathbf{0} \\
\mathbf{0} & \mathbf{Z}(\tau)
\end{array}\right]\left[\begin{array}{c}
\mathbf{T}_{(L)} \\
\mathbf{T}_{(P)}
\end{array}\right] \mathbf{R}_{2}(\tau) \mathrm{d} \tau,
\end{aligned}
$$

$$
\begin{aligned}
& \mathbf{a}_{(A)}(t) \\
& \quad=\int_{0}^{t} \ddot{\mathbf{H}}^{(A)}(t-\tau)\left[\begin{array}{cc}
\mathbf{Z}(\tau) & \mathbf{0} \\
\mathbf{0} & \mathbf{Z}(\tau)
\end{array}\right]\left[\begin{array}{l}
\mathbf{T}_{(L)} \\
\mathbf{T}_{(P)}
\end{array}\right] \mathbf{R}_{2}(\tau) \mathrm{d} \tau,
\end{aligned}
$$




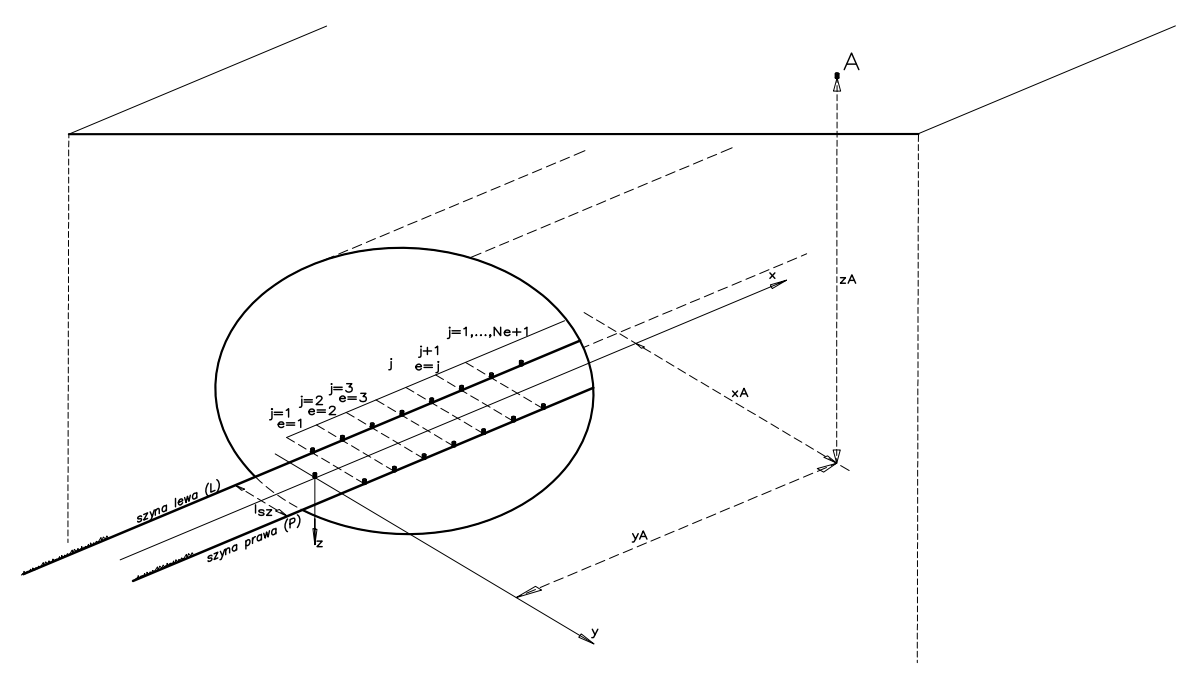

Fig. 2. Discretization of the vehicle path.

where $\mathbf{T}_{(L)}$ and $\mathbf{T}_{(P)}$ are transformation matrices, $\mathbf{Z}(t)$ is a non-stationary matrix describing the contact point motion along the railhead. The solution for the displacements in Eqn. 23) and the calculation of derivatives are performed numerically, and for every step $n, \mathbf{x}_{2}^{(n)}=$ $\mathbf{x}_{N}^{(n)}+\mathbf{u}_{2 t}^{(n)}$, and $\mathbf{u}_{2 t}^{(n)}$ is equal to

$$
\begin{aligned}
\mathbf{u}_{2 t}^{(n)}= & \mathrm{d} T\left[\begin{array}{l}
\mathbf{T}_{(L)} \\
\mathbf{T}_{(P)}
\end{array}\right]^{T}\left[\begin{array}{cc}
\mathbf{Z}^{(n)} & \mathbf{0} \\
\mathbf{0} & \mathbf{Z}^{(n)}
\end{array}\right]^{T} \\
& \times \sum_{m=1}^{n} \mathbf{H}^{(n-m+1)}\left[\begin{array}{cc}
\mathbf{Z}^{(m)} \mathbf{0} \\
\mathbf{0} & \mathbf{Z}^{(m)}
\end{array}\right]\left[\begin{array}{l}
\mathbf{T}_{(L)} \\
\mathbf{T}_{(P)}
\end{array}\right] \mathbf{R}_{2}^{(m)}
\end{aligned}
$$

Writing

$$
\begin{gathered}
\mathbf{Y}^{(n)}=\left[\begin{array}{cc}
\mathbf{Z}^{(n)} & \mathbf{0} \\
\mathbf{0} & \mathbf{Z}^{(n)}
\end{array}\right]\left[\begin{array}{l}
\mathbf{T}_{(L)} \\
\mathbf{T}_{(P)}
\end{array}\right], \\
\dot{\mathbf{Y}}^{(n)}=\left[\begin{array}{cc}
\dot{\mathbf{Z}}^{(n)} & \mathbf{0} \\
\mathbf{0} & \dot{\mathbf{Z}}^{(n)}
\end{array}\right]\left[\begin{array}{c}
\mathbf{T}_{(L)} \\
\mathbf{T}_{(P)}
\end{array}\right], \\
\ddot{\mathbf{Y}}^{(n)}=\left[\begin{array}{cc}
\ddot{\mathbf{Z}}^{(n)} & \mathbf{0} \\
\mathbf{0} & \ddot{\mathbf{Z}}^{(n)}
\end{array}\right]\left[\begin{array}{c}
\mathbf{T}_{(L)} \\
\mathbf{T}_{(P)}
\end{array}\right],
\end{gathered}
$$

the solution for $\mathbf{u}_{2 t}^{(n)}$ at every step is

$$
\mathbf{u}_{2 t}^{(n)}=\mathbf{A}^{(n)}+\mathbf{B}^{(n)} \mathbf{R}_{2}^{(n)}
$$

with $\mathbf{A}^{(1)}=\mathbf{0}$, and

$$
\mathbf{A}^{(n)}=\mathrm{d} T \mathbf{Y}^{(n) T} \sum_{m=1}^{n-1} \mathbf{H}^{(n-m+1)} \mathbf{Y}^{(m)} \mathbf{R}_{2}^{(m)},
$$

$$
n=2, \ldots, N_{T}
$$

and

$$
\mathbf{B}^{(n)}=\mathrm{d} T \mathbf{Y}^{(n) T} \mathbf{H}^{(1)} \mathbf{Y}^{(n)}, \quad n=1, \ldots, N_{T} .
$$

The first-order derivative with respect to time is

$$
\dot{\mathbf{u}}_{2 t}^{(n)}=\dot{\mathbf{A}}^{(n)}+\dot{\mathbf{B}}^{(n)} \mathbf{R}_{2}^{(n)},
$$

where $\dot{\mathbf{A}}^{(1)}=\mathbf{0}$, and

$$
\begin{aligned}
\dot{\mathbf{A}}^{(n)}= & \mathrm{d} T \sum_{m=1}^{n-1}\left(\dot{\mathbf{Y}}^{(n) T} \mathbf{H}^{(n-m+1)}\right. \\
+ & \left.\mathbf{Y}^{(n) T} \dot{\mathbf{H}}^{(n-m+1)}\right) \mathbf{Y}^{(m)} \mathbf{R}_{2}^{(m)}, \\
& n=2, \ldots, N_{T}
\end{aligned}
$$

and

$$
\begin{array}{r}
\dot{\mathbf{B}}^{(n)}=\mathrm{d} T\left(\dot{\mathbf{Y}}^{(n) T} \mathbf{H}^{(1)}+\mathbf{Y}^{(n) T} \dot{\mathbf{H}}^{(1)}\right) \mathbf{Y}^{(n)} \\
n=1, \ldots, N_{T} .
\end{array}
$$

The second-order derivative with respect to time is

$$
\ddot{\mathbf{u}}_{2 t}^{(n)}=\ddot{\mathbf{A}}^{(n)}+\ddot{\mathbf{B}}^{(n)} \mathbf{R}_{2}^{(n)},
$$

where $\ddot{\mathbf{A}}^{(1)}=\mathbf{0}$, and

$$
\begin{aligned}
\ddot{\mathbf{A}}^{(n)}= & \mathrm{d} T \sum_{m=1}^{n-1}\left[\ddot{\mathbf{Y}}^{(n) T} \mathbf{H}^{(n-m+1)}\right. \\
& \left.+2 \dot{\mathbf{Y}}^{(n) T} \dot{\mathbf{H}}^{(n-m+1)}+\mathbf{Y}^{(n) T} \ddot{\mathbf{H}}^{(n-m+1)}\right] \\
& \times \mathbf{Y}^{(m)} \mathbf{R}_{2}^{(m)}, \quad n=2, \ldots, N_{T}
\end{aligned}
$$


and

$$
\begin{aligned}
\ddot{\mathbf{B}}^{(n)}=\mathrm{d} T & \left(\ddot{\mathbf{Y}}^{(n) T} \mathbf{H}^{(1)}+2 \dot{\mathbf{Y}}^{(n) T} \dot{\mathbf{H}}^{(1)}\right. \\
& \left.+\mathbf{Y}^{(n) T} \ddot{\mathbf{H}}^{(1)}\right) \mathbf{Y}^{(n)}, \quad n=1, \ldots, N_{T}
\end{aligned}
$$

$\mathbf{A}^{(n)}$ is the convolution matrix of up to the $(n-1)$ th step, while $\mathbf{B}^{(n)}$ is the convolution matrix at the current step $n$. Both matrices have their time derivatives, $\dot{\mathbf{A}}^{(n)}, \ddot{\mathbf{A}}^{(n)}, \dot{\mathbf{B}}^{(n)}, \ddot{\mathbf{B}}^{(n)}$.

The equation of motion is then modified as follows:

$$
\left\{\begin{aligned}
\mathbf{M}_{11} \ddot{\mathbf{x}}_{1}^{(n)}+\mathbf{C}_{11} \dot{\mathbf{x}}_{1}^{(n)}+\mathbf{K}_{11} \mathbf{x}_{1}^{(n)} & \mathbf{Q}_{1}^{(n)}+\mathbf{P}_{1}^{(n)}-\left(\mathbf{M}_{12} \ddot{\mathbf{x}}_{N}^{(n)}+\mathbf{C}_{12} \dot{\mathbf{x}}_{N}^{(n)}+\mathbf{K}_{12} \mathbf{x}_{N}^{(n)}\right) \\
& -\left(\mathbf{M}_{12} \ddot{\mathbf{A}}^{(n)}+\mathbf{C}_{12} \dot{\mathbf{A}}^{(n)}+\mathbf{K}_{12} \mathbf{A}^{(n)}\right) \\
& -\left(\mathbf{M}_{12} \ddot{\mathbf{B}}^{(n)}+\mathbf{C}_{12} \dot{\mathbf{B}}^{(n)}+\mathbf{K}_{12} \mathbf{B}^{(n)}\right) \mathbf{R}_{2}^{(n)} \\
\mathbf{R}_{2}^{(n)} & =\mathbf{Q}_{2}^{(n)}+\mathbf{P}_{2}^{(n)} \\
& -\left(\mathbf{M}_{21} \ddot{\mathbf{x}}_{1}^{(n)}+\mathbf{C}_{21} \dot{\mathbf{x}}_{1}^{(n)}+\mathbf{K}_{21} \mathbf{x}_{1}^{(n)}\right) \\
& -\left(\mathbf{M}_{22} \ddot{\mathbf{x}}_{N}^{(n)}+\mathbf{C}_{22} \dot{\mathbf{x}}_{N}^{(n)}+\mathbf{K}_{22} \mathbf{x}_{N}^{(n)}\right) \\
& -\left(\mathbf{M}_{22} \ddot{\mathbf{A}}^{(n)}+\mathbf{C}_{22} \dot{\mathbf{A}}^{(n)}+\mathbf{K}_{22} \mathbf{A}^{(n)}\right) \\
& -\left(\mathbf{M}_{22} \ddot{\mathbf{B}}^{(n)}+\mathbf{C}_{22} \dot{\mathbf{B}}^{(n)}+\mathbf{K}_{22} \mathbf{B}^{(n)}\right) \mathbf{R}_{2}^{(n)}
\end{aligned}\right.
$$

and further re-arranged as

$$
\left\{\begin{aligned}
\mathbf{M}_{11} \ddot{\mathbf{x}}_{1}^{(n)}+\mathbf{C}_{11} \dot{\mathbf{x}}_{1}^{(n)}+\mathbf{K}_{11} \mathbf{x}_{1}^{(n)} \\
=\mathbf{Q}_{1}^{(n)}+\mathbf{P}_{1}^{(n)}-\left(\mathbf{M}_{12} \ddot{\mathbf{x}}_{N}^{(n)}+\mathbf{C}_{12} \dot{\mathbf{x}}_{N}^{(n)}+\mathbf{K}_{12} \mathbf{x}_{N}^{(n)}\right) \\
\quad-\left(\mathbf{M}_{12} \ddot{\mathbf{A}}^{(n)}+\mathbf{C}_{12} \dot{\mathbf{A}}^{(n)}+\mathbf{K}_{12} \mathbf{A}^{(n)}\right) \\
\quad-\left(\mathbf{M}_{12} \ddot{\mathbf{B}}^{(n)}+\mathbf{C}_{12} \dot{\mathbf{B}}^{(n)}+\mathbf{K}_{12} \mathbf{B}^{(n)}\right) \mathbf{R}_{2}^{(n)}, \\
\left(\mathbf{I}+\mathbf{M}_{22} \ddot{\mathbf{B}}^{(n)}+\mathbf{C}_{22} \dot{\mathbf{B}}^{(n)}+\mathbf{K}_{22} \mathbf{B}^{(n)}\right) \mathbf{R}_{2}^{(n)} \\
=\mathbf{Q}_{2}^{(n)}+\mathbf{P}_{2}^{(n)}-\left(\mathbf{M}_{21} \ddot{\mathbf{x}}_{1}^{(n)}+\mathbf{C}_{21} \dot{\mathbf{x}}_{1}^{(n)}+\mathbf{K}_{21} \mathbf{x}_{1}^{(n)}\right) \\
\quad-\left(\mathbf{M}_{22} \ddot{\mathbf{x}}_{N}^{(n)}+\mathbf{C}_{22} \dot{\mathbf{x}}_{N}^{(n)}+\mathbf{K}_{22} \mathbf{x}_{N}^{(n)}\right) \\
\quad-\left(\mathbf{M}_{22} \ddot{\mathbf{A}}^{(n)}+\mathbf{C}_{22} \dot{\mathbf{A}}^{(n)}+\mathbf{K}_{22} \mathbf{A}^{(n)}\right) .
\end{aligned}\right.
$$

Now, the following matrices can be defined:

$$
\begin{aligned}
& \mathbf{G}_{12}^{(n)}=\mathbf{M}_{12} \ddot{\mathbf{x}}_{N}^{(n)}+\mathbf{C}_{12} \dot{\mathbf{x}}_{N}^{(n)}+\mathbf{K}_{12} \mathbf{x}_{N}^{(n)}, \\
& \mathbf{G}_{22}^{(n)}=\mathbf{M}_{22} \ddot{\mathbf{x}}_{N}^{(n)}+\mathbf{C}_{22} \dot{\mathbf{x}}_{N}^{(n)}+\mathbf{K}_{22} \mathbf{x}_{N}^{(n)} .
\end{aligned}
$$

$\mathbf{G}_{12}^{(n)}$ and $\mathbf{G}_{22}^{(n)}$ are the dynamic forces due to unevenness at the current location along the rail. We have

$$
\mathbf{D}_{12}^{(n)}=\mathbf{M}_{12} \ddot{\mathbf{A}}^{(n)}+\mathbf{C}_{12} \dot{\mathbf{A}}^{(n)}+\mathbf{K}_{12} \mathbf{A}^{(n)},
$$

$$
\mathbf{D}_{22}^{(n)}=\mathbf{M}_{22} \ddot{\mathbf{A}}^{(n)}+\mathbf{C}_{22} \dot{\mathbf{A}}^{(n)}+\mathbf{K}_{22} \mathbf{A}^{(n)} .
$$

$\mathbf{D}_{12}^{(n)}$ and $\mathbf{D}_{22}^{(n)}$ are the dynamic subgrade reactions due to the loading history, dependent only on the convolution matrix $\mathbf{A}^{(n)}$ and its time derivatives,

$$
\begin{aligned}
& \mathbf{W}_{12}^{(n)}=\mathbf{M}_{12} \ddot{\mathbf{B}}^{(n)}+\mathbf{C}_{12} \dot{\mathbf{B}}^{(n)}+\mathbf{K}_{12} \mathbf{B}^{(n)}, \\
& \mathbf{W}_{22}^{(n)}=\mathbf{I}+\mathbf{M}_{22} \ddot{\mathbf{B}}^{(n)}+\mathbf{C}_{22} \dot{\mathbf{B}}^{(n)}+\mathbf{K}_{22} \mathbf{B}^{(n)} .
\end{aligned}
$$

$\mathbf{W}_{12}^{(n)}$ and $\mathbf{W}_{22}^{(n)}$ are the current dynamic subgrade reactions, dependent only on the current convolution matrix $\mathbf{B}^{(n)}$ and its time derivatives. Writing the total forces as

$$
\begin{aligned}
& \mathbf{F}_{12}^{(n)}=\mathbf{Q}_{1}^{(n)}+\mathbf{P}_{1}^{(n)}-\mathbf{G}_{12}^{(n)}-\mathbf{D}_{12}^{(n)}, \\
& \mathbf{F}_{22}^{(n)}=\mathbf{Q}_{2}^{(n)}+\mathbf{P}_{2}^{(n)}-\mathbf{G}_{22}^{(n)}-\mathbf{D}_{22}^{(n)},
\end{aligned}
$$

the equation of motion can be given as

$$
\left\{\begin{array}{l}
\mathbf{M}_{11} \ddot{\mathbf{x}}_{1}^{(n)}+\mathbf{C}_{11} \dot{\mathbf{x}}_{1}^{(n)}+\mathbf{K}_{11} \mathbf{x}_{1}^{(n)}=\mathbf{F}_{12}^{(n)}-\mathbf{W}_{12}^{(n)} \mathbf{R}_{2}^{(n)}, \\
\mathbf{W}_{22}^{(n)} \mathbf{R}_{2}^{(n)}=\mathbf{F}_{22}^{(n)} \\
\quad-\left(\mathbf{M}_{21} \ddot{\mathbf{x}}_{1}^{(n)}+\mathbf{C}_{21} \dot{\mathbf{x}}_{1}^{(n)}+\mathbf{K}_{21} \mathbf{x}_{1}^{(n)}\right),
\end{array}\right.
$$

when $\mathbf{W}_{0}^{(n)}=\mathbf{W}_{12}^{(n)} \mathbf{W}_{22}^{(n)-1}$. Equation (32) yields

$$
\left\{\begin{array}{l}
\mathbf{M}_{11} \ddot{\mathbf{x}}_{1}^{(n)}+\mathbf{C}_{11} \dot{\mathbf{x}}_{1}^{(n)}+\mathbf{K}_{11} \mathbf{x}_{1}^{(n)}=\mathbf{F}_{12}^{(n)} \\
\mathbf{W}_{0}^{(n)} \mathbf{F}_{22}^{(n)}+\mathbf{W}_{0}^{(n)}\left(\mathbf{M}_{21} \ddot{\mathbf{x}}_{1}^{(n)}+\mathbf{C}_{21} \dot{\mathbf{x}}_{1}^{(n)}+\mathbf{K}_{21} \mathbf{x}_{1}^{(n)}\right), \\
\mathbf{R}_{2}^{(n)}=\mathbf{W}_{22}^{(n)-1} \mathbf{F}_{22}^{(n)} \\
\quad-\mathbf{W}_{22}^{(n)-1}\left(\mathbf{M}_{21} \ddot{\mathbf{x}}_{1}^{(n)}+\mathbf{C}_{21} \dot{\mathbf{x}}_{1}^{(n)}+\mathbf{K}_{21} \mathbf{x}_{1}^{(n)}\right) .
\end{array}\right.
$$

Substituting

$$
\begin{gathered}
\mathbf{M}_{0}^{(n)}=\mathbf{M}_{11}-\mathbf{W}_{0}^{(n)} \mathbf{M}_{21}, \\
\mathbf{C}_{0}^{(n)}=\mathbf{C}_{11}-\mathbf{W}_{0}^{(n)} \mathbf{C}_{21}, \\
\mathbf{K}_{0}^{(n)}=\mathbf{K}_{11}-\mathbf{W}_{0}^{(n)} \mathbf{K}_{21}, \\
\mathbf{F}^{(n)}=\mathbf{F}_{12}^{(n)}-\mathbf{W}_{0}^{(n)} \mathbf{F}_{22}^{(n)},
\end{gathered}
$$

one may compute the solution for the central differences:

$$
\left\{\begin{array}{l}
\mathbf{M}_{0}^{(n)} \ddot{\mathbf{x}}_{1}^{(n)}+\mathbf{C}_{0}^{(n)} \dot{\mathbf{x}}_{1}^{(n)}+\mathbf{K}_{0}^{(n)} \mathbf{x}_{1}^{(n)}=\mathbf{F}^{(n)} \\
\mathbf{R}_{2}^{(n)}=\mathbf{W}_{22}^{(n)-1} \\
\times\left[\mathbf{F}_{22}^{(n)}-\left(\mathbf{M}_{21} \ddot{\mathbf{x}}_{1}^{(n)}+\mathbf{C}_{21} \dot{\mathbf{x}}_{1}^{(n)}+\mathbf{K}_{21} \mathbf{x}_{1}^{(n)}\right)\right]
\end{array}\right.
$$

where $\mathbf{F}^{(n)}$ is the force matrix independent of $\mathbf{R}_{2}^{(n)}$. $\mathbf{M}_{0}^{(n)}, \mathbf{C}_{0}^{(n)}, \mathbf{K}_{0}^{(n)}$ are non-stationary matrices of the vehicle system due to the movement of inertial loading.

Accelerations

$$
\ddot{\mathbf{x}}_{1}^{(n)}=\frac{\mathbf{x}_{1}^{(n+1)}-2 \mathbf{x}_{1}^{(n)}+\mathbf{x}_{1}^{(n-1)}}{\mathrm{d} T^{2}},
$$


and velocities

$$
\dot{\mathbf{x}}_{1}^{(n)}=\frac{\mathbf{x}_{1}^{(n+1)}-\mathbf{x}_{1}^{(n-1)}}{2 \mathrm{~d} T},
$$

$n=1, \ldots, N_{T}$, fulfil the initial conditions

$$
\begin{aligned}
& \mathbf{x}_{1}^{(1)}=\mathbf{x}(0)=\mathbf{u}_{0}, \\
& \dot{\mathbf{x}}_{1}^{(1)}=\dot{\mathbf{x}}(0)=\mathbf{v}_{0},
\end{aligned}
$$

and

$$
\mathbf{x}_{1}^{(2)}-\mathbf{x}_{1}^{(0)}=2 \mathrm{~d} T \mathbf{v}_{0} .
$$

Hence

$$
\begin{aligned}
\mathbf{x}_{1}^{(2)} & -2 \mathbf{u}_{0}+\mathbf{x}_{1}^{(0)} \\
& =\mathrm{d} T^{2} \mathbf{M}_{0}^{(1)-1}\left(\mathbf{F}^{(1)}-\mathbf{C}_{0}^{(1)} \mathbf{v}_{0}-\mathbf{K}_{0}^{(1)} \mathbf{u}_{0}\right),
\end{aligned}
$$

and

$$
\begin{aligned}
\mathbf{x}_{1}^{(2)}= & \mathbf{u}_{0}+\mathrm{d} T \mathbf{v}_{0} \\
& +\frac{\mathrm{d} T^{2}}{2} \mathbf{M}_{0}^{(1)-1}\left(\mathbf{F}^{(1)}-\mathbf{C}_{0}^{(1)} \mathbf{v}_{0}-\mathbf{K}_{0}^{(1)} \mathbf{u}_{0}\right) .
\end{aligned}
$$

If $\mathbf{v}_{0}=\mathbf{0}$, then

$$
\mathbf{x}_{1}^{(2)}=\mathbf{u}_{0}+\frac{d T^{2}}{2} \mathbf{M}_{0}^{(1)-1}\left(\mathbf{F}^{(1)}-\mathbf{K}_{0}^{(1)} \mathbf{u}_{0}\right)
$$

and

$$
\begin{aligned}
\mathbf{M}_{0}^{(n)} & \frac{\mathbf{x}_{1}^{(n+1)}-2 \mathbf{x}_{1}^{(n)}+\mathbf{x}_{1}^{(n-1)}}{\mathrm{d} T^{2}} \\
& +\mathbf{C}_{0}^{(n)} \frac{\mathbf{x}_{1}^{(n+1)}-\mathbf{x}_{1}^{(n-1)}}{2 \mathrm{~d} T}+\mathbf{K}_{0}^{(n)} \mathbf{x}_{1}^{(n)}=\mathbf{F}^{(n)}
\end{aligned}
$$

for steps $n=2, \ldots, N_{T}$,

$$
\begin{aligned}
\left(\frac{1}{\mathrm{~d} T}\right. & \left.\mathbf{M}_{0}^{(n)}+\frac{1}{2} \mathbf{C}_{0}^{(n)}\right) \mathbf{x}_{1}^{(n+1)} \\
= & \mathrm{d} T \mathbf{F}^{(n)}+\left(\frac{2}{\mathrm{~d} T} \mathbf{M}_{0}^{(n)}-\mathrm{d} T \mathbf{K}_{0}^{(n)}\right) \mathbf{x}_{1}^{(n)} \\
& +\left(\frac{1}{2} \mathbf{C}_{0}^{(n)}-\frac{1}{\mathrm{~d} T} \mathbf{M}_{0}^{(n)}\right) \mathbf{x}_{1}^{(n-1)} .
\end{aligned}
$$

Applying

$$
\begin{aligned}
\mathbf{S}_{M C}^{(n)} & =\frac{1}{\mathrm{~d} T} \mathbf{M}_{0}^{(n)}+\frac{1}{2} \mathbf{C}_{0}^{(n)} \\
\mathbf{S}_{M K}^{(n)} & =\frac{2}{\mathrm{~d} T} \mathbf{M}_{0}^{(n)}-\mathrm{d} T \mathbf{K}_{0}^{(n)}, \\
\mathbf{S}_{C M}^{(n)} & =\frac{1}{2} \mathbf{C}_{0}^{(n)}-\frac{1}{\mathrm{~d} T} \mathbf{M}_{0}^{(n)}
\end{aligned}
$$

one gets the recurrent formula

$$
\mathbf{S}_{M C}^{(n)} \mathbf{x}_{1}^{(n+1)}=\mathrm{d} T \mathbf{F}^{(n)}+\mathbf{S}_{M K}^{(n)} \mathbf{x}_{1}^{(n)}+\mathbf{S}_{C M}^{(n)} \mathbf{x}_{1}^{(n-1)} .
$$

A further reformulation of Eqn. (14),

$$
\begin{aligned}
\mathbf{W}_{22}^{(n)} \mathbf{R}_{2}^{(n)}= & \mathbf{F}_{22}^{(n)}-\mathbf{M}_{21} \frac{\mathbf{x}_{1}^{(n+1)}-2 \mathbf{x}_{1}^{(n)}+\mathbf{x}_{1}^{(n-1)}}{\mathrm{d} T^{2}} \\
& -\mathbf{C}_{21} \frac{\mathbf{x}_{1}^{(n+1)}-\mathbf{x}_{1}^{(n-1)}}{2 \mathrm{~d} T}-\mathbf{K}_{21} \mathbf{x}_{1}^{(n)}
\end{aligned}
$$

gives another recurrent formula:

$$
\begin{aligned}
\mathbf{W}_{22}^{(n)} & \mathbf{R}_{2}^{(n)} \\
= & \mathbf{F}_{22}^{(n)}-\frac{1}{\mathrm{~d} T}\left[\left(\frac{1}{\mathrm{~d} T} \mathbf{M}_{21}+\frac{1}{2} \mathbf{C}_{21}\right) \mathbf{x}_{1}^{(n+1)}\right. \\
& +\left(\mathrm{d} T \mathbf{K}_{21}-\frac{2}{\mathrm{~d} T} \mathbf{M}_{21}\right) \mathbf{x}_{1}^{(n)} \\
& \left.+\left(\frac{1}{\mathrm{~d} T} \mathbf{M}_{21}-\frac{1}{2} \mathbf{C}_{21}\right) \mathbf{x}_{1}^{(n-1)}\right]
\end{aligned}
$$

\section{Numerical predictions}

The computations of reaction $\mathbf{R}_{2}^{(n)}$ at every step have to use the value of displacement $\mathbf{x}_{1}^{(n+1)}$. At first, a sequential Matlab code was generated. The package installed on SunFire-6800 at Cracow ACC Cyfronet was used.

Figure 3 shows the time history and frequency content of the free field acceleration for the passage of a typical single tram vehicle N105a running on a track built on the surface. The track is also a typical slab track with identified dynamic parameters. The kinematic excitation is different for both rails and every single wheel. At a distance $x=8 \mathrm{~m}$ from the track, the passage of an individual bogie cannot be identified from the time history of acceleration, while at $x=24 \mathrm{~m}$ both bogies are visible in the time history. Frequencies higher than $20 \mathrm{~Hz}$ are attenuated mostly by the material and radiation damping in the soil at $x=24 \mathrm{~m}$ from the track.

Figure 4 presents the time history and frequency content of the free field velocity as computed for the passage of the same single tram vehicle N105a. At a distance $x=8 \mathrm{~m}$ from the track, the passage of an individual bogie may be seen at the time history of velocity. The lower frequencies are visible better in the velocity frequency content than in the acceleration frequency content. Figure 5 shows the trajectories of the free field velocity as computed for the soil particles during the passage of the tram moving at $v=40 \mathrm{~km} / \mathrm{h}$ at $x=8 \mathrm{~m}$ and $x=24 \mathrm{~m}$ from the track. The trajectories present the behaviour of a single soil particle in space. At $x=8 \mathrm{~m}$ from the track (Fig. 5(a)), soil particle vibration is rather chaotic, while at $x=24 \mathrm{~m}$ from the track (Fig. 5 b)), the particle movement is mostly in the horizontal direction. 


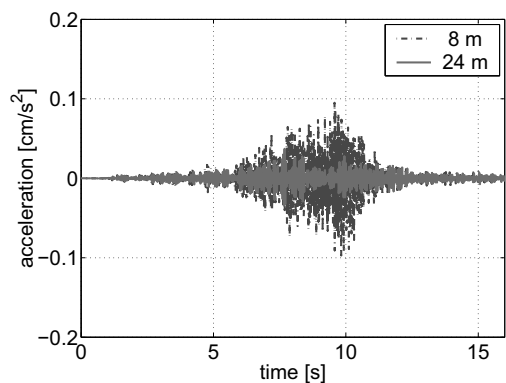

(a)

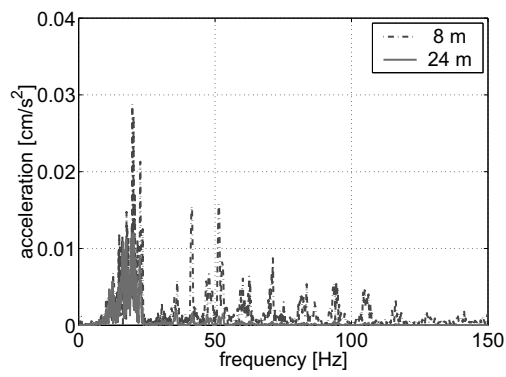

(b)

Fig. 3. Time history (a) and frequency content (b) of vertical soil acceleration during the passage of a tram moving at $v=40 \mathrm{~km} / \mathrm{h}$ at $x=8 \mathrm{~m}$ and $x=24 \mathrm{~m}$ from the track.

\section{Parallel version of the computational model}

As already mentioned, at first the sequential Matlab code was generated. Later on, the numerical formulation was modified as a significant reduction in calculation time was expected, using high-performance computers. Figure 6 presents a parallel computation scheme of dynamic updating matrices at every discrete step.

At the beginning, the dynamic forces $\mathbf{G}_{12}^{(n)}, \mathbf{G}_{22}^{(n)}$ due to the unevenness at current position along the rail may be computed. In the same step, the convolution matrix $\mathbf{A}^{(n)}$ and its time derivatives, as well as the current convolution matrix $\mathbf{B}^{(n)}$ and its time derivatives may be computed in parallel. In the next step, the convolution matrix $\mathbf{A}^{(n)}$ and its time derivatives are needed to compute the dynamic subgrade reactions to the loading history $\mathbf{D}_{12}^{(n)}, \mathbf{D}_{22}^{(n)}$, while the current convolution matrix $\mathbf{B}^{(n)}$ and its time derivatives are needed independently to compute current dynamic subgrade reactions $\mathbf{W}_{12}^{(n)}, \mathbf{W}_{22}^{(n)}$ and, afterwards, their combination $\mathbf{W}_{0}^{(n)}$. Having the dynamic forces due to the unevenness $\mathbf{G}_{12}^{(n)}, \mathbf{G}_{22}^{(n)}$ and the dynamic subgrade reactions $\mathbf{D}_{12}^{(n)}, \mathbf{D}_{22}^{(n)}$, total forces $\mathbf{F}_{12}^{(n)}, \mathbf{F}_{22}^{(n)}$ are computed. Here, the loops of the parallel computations meet at the critical point. The second loop starts with the computation of dynamic force $\mathbf{F}^{(n)}$ and, in parallel, non-stationary matrices of the vehicle system $\mathbf{M}_{0}^{(n)}, \mathbf{C}_{0}^{(n)}, \mathbf{K}_{0}^{(n)}$. Using these matrices, $\mathbf{S}_{M C}^{(n)}, \mathbf{S}_{M K}^{(n)}, \mathbf{S}_{C M}^{(n)}$ are computed. Finally, the next step

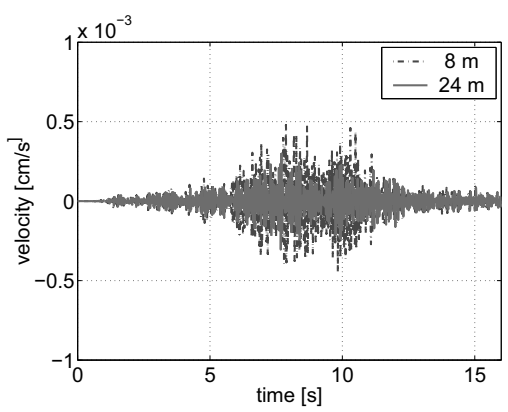

(a)

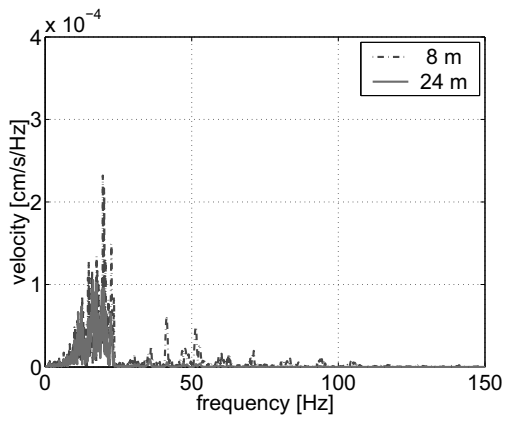

(b)

Fig. 4. Time history (a) and frequency content (b) of vertical soil velocity during the passage of a tram moving at $v=$ $40 \mathrm{~km} / \mathrm{h}$ at $x=8 \mathrm{~m}$ and $x=24 \mathrm{~m}$ from the track.

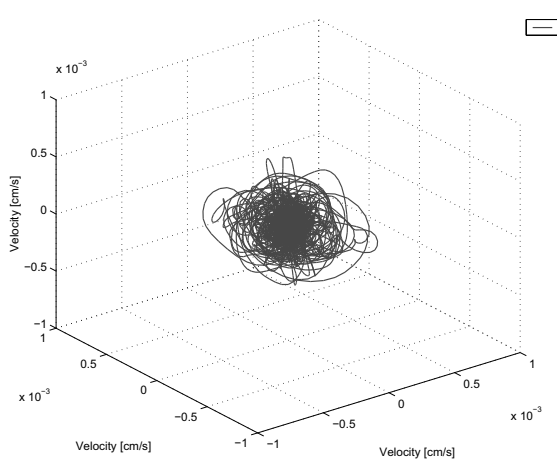

(a)

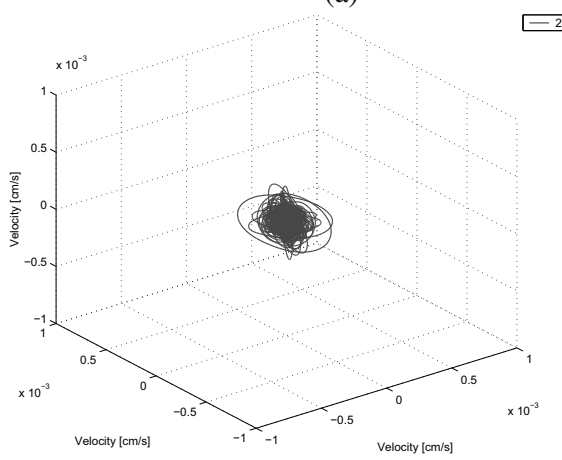

(b)

Fig. 5. Trajectories of soil particle velocity during the passage of the tram with a speed of $v=40 \mathrm{~km} / \mathrm{h}$ at $x=8 \mathrm{~m}$ (a) and $x=24 \mathrm{~m}$ (b) from the track. 


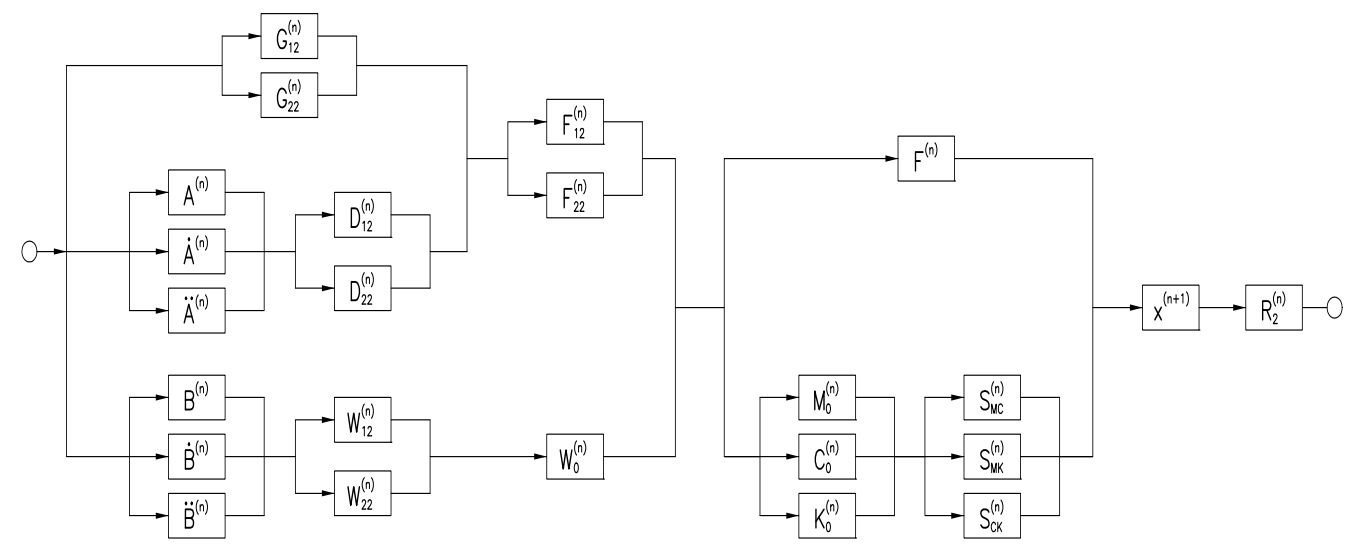

Fig. 6. Idea of the parallelization of model computations.

displacement $\mathbf{x}^{(n+1)}$ is computed. Having that, the dynamic reaction $\mathbf{R}_{2}^{(n)}$ is known.

The Message Passing Interface (MPI) library or OpenMP Compiler Directives may be used as alternative parallelization tools of the code parallelize the loops shown in Fig. 6 .

\section{Final remarks}

The prediction model of vibrations due to railway traffic is formulated in this paper. A vehicle running on a track is excited by wheel and rail irregularities, as well as track vibration due to the vehicle movement. Dynamic forces are generated in such a way by the train. The free field response is a convolution of the fundamental solution, and the dynamic forces and may be computed numerically with parallel modifications. The vehicle parameters as well as the parameters of the track and soil may be identified. The entire model may be validated by means of "in-situ" measurements on several levels including track (tunnel) and soil vibrations.

\section{References}

Cox, S. and Wang, A. (2003). Effect of track stiffness on vibration levels in railway tunnels, Journal of Sound and Vibration 267(3): 565-573.

Gupta, S., Hussein, M., Degrande, G., Hunt, H. and Clouteau, D. (2007). A comparison of two numerical models for the prediction of vibrations from underground railway traffic, Soil Dynamics and Earthquake Engineering 27(7): 608-624.

Kogut, J. and Ciurej, H. (2005a). Numerical modelling of the train-track axle forces, Mechanika 222(65): 209-218.

Kogut, J. and Ciurej, H. (2005b). The numerical study of the dynamic train axle forces induced on an uneven railway track, Czasopismo Techniczne 102(12-B): 35-49.
Kogut, J. and Ciurej, H. (2006). A numerical modelling and an identification of the parameters of the tramway bogie, in T. Uhl (Ed.), Selected Problems of Modal Analysis of Mechnical Constructions, Institute for Sustainable Technologies-National Research Institute, Cracow, pp. 156-164.

Kogut, J. and Ciurej, H. (2008). A numerical model formulation for underground railway vibration prediction, Mechanika 258(74): 143-154.

Steenbergen, M. (2008). Quantification of dynamic wheel-rail contact forces at short rail irregularities and application to measured rail welds, Journal of Sound and Vibration 312(4-5): 606-629.

Van den Broeck, P. (2001). A prediction model for ground-borne vibrations due to railway traffic, Ph.D. thesis, Department of Civil Engineering, KU Leuven, Leuven.

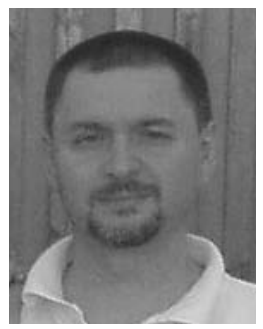

Janusz P. Kogut holds his Ph.D. in structura mechanics and has been granted a post-doctoral position at KU Leuven. He works at the SoilStructure Interaction Division, Civil Engineering Department, Cracow University of Technology. His main area of interest covers artificial intelligence and its application to advanced civil engineering problems.

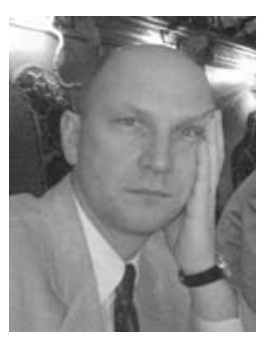

Henryk Ciurej holds his Ph.D. in structural dynamics and works at the Institute of Structural Mechanics, Civil Engineering Department, Cracow University of Technology. He is also a senior partner in CG Structural Consulting. He is mostly interested in numerical modeling, structural optimization and identification problems.

Received: 12 June 2009

Revised: 17 November 2009

Re-revised: 13 January 2010 